\title{
Photoinduced birefringence of azobenzene polymer at blue excitation wavelengths
}

\author{
Anna Kozanecka-Szmigiel • Krzysztof Switkowski • \\ Ewa Schab-Balcerzak · Dariusz Szmigiel
}

Received: 4 August 2014 / Accepted: 5 February 2015 / Published online: 26 February 2015

(c) The Author(s) 2015. This article is published with open access at Springerlink.com

\begin{abstract}
The photoinduced birefringence resulting from trans-cis-trans isomerizations has been measured in a thin film of azobenzene-functionalized poly(esterimide) at three excitation wavelengths. The excitation wavelengths of 388 and $420 \mathrm{~nm}$ have been located at exactly opposite sides of the trans azobenzene absorption band. A third irradiation wavelength was shifted to $438 \mathrm{~nm}$. Distinct saturation levels and growth dynamics of photoinduced birefringence have been observed under the 388- and 420-nm illumination, while very similar characteristics were recorded at 420 and $438 \mathrm{~nm}$. Differences in the obtained birefringence signals have been explained considering the cis isomer absorption and photoisomerization pathways.
\end{abstract}

\section{Introduction}

Azobenzene polymers contain the azobenzene groups or their derivatives either as dopants or incorporated into the backbone. They possess exceptional potential for technological applications in high-density data storage, optical switching, diffractive optics and photomechanics [1-7] due to the unique photoinduced effects they may exhibit: the generation of optical anisotropy or surface relief gratings

A. Kozanecka-Szmigiel $(\bowtie) \cdot$ K. Switkowski

Faculty of Physics, Warsaw University of Technology, 75

Koszykowa Str., 00-662 Warsaw, Poland

e-mail: annak@if.pw.edu.pl

E. Schab-Balcerzak

Centre of Polymer and Carbon Materials, Polish Academy

of Sciences, M. Curie-Sklodowska 34, 41-819 Zabrze, Poland

D. Szmigiel

Institute of Electron Technology, al. Lotnikow 32/46,

02-668 Warsaw, Poland and photomechanical deformation of thin films. The materials photoresponsive behavior arises from the presence of the photochromic azobenzene molecules [8]. These molecules can exist in two isomeric forms, which may be reversibly interconverted between each other by interaction with light of a proper wavelength. The cis-to-trans conversion may be induced thermally as well. A linearly polarized irradiation of a proper wavelength may induce optical anisotropy in the polymer material, i.e., optical dichroism and birefringence appearing as an increased absorption and refractive index in a direction perpendicular to the polarization of the excitation light, respectively. This optical anisotropy arises from an orientational order of azochromophores $[9,10]$. The order is generated as a result of angular selectivity of absorption events by highly anisotropic trans molecules and of the trans-cis reversible isomerization. The rotation of the photochromic molecules can occur during the photochemical reaction (trans-cis or cis-trans) or during the lifetime of the cis molecule since the rotational diffusion of this more spherical isomer is more efficient than in the case of the trans molecule [11]. After multiple isomerization cycles, the azochromophores finally align in one of the directions perpendicular to the laser polarization, i.e., in the position, for which the light is no longer absorbed by the molecules.

The photoinduced birefringence level and growth rate in azobenzene polymers depend on numerous factors referring to the chromophore and polymer architecture (e.g., substituents on the azobenzene group, polymer backbone, type of linkage between the chromophore and the main chain) but also to the illumination conditions (e.g., excitation wavelength or intensity). A lot of studies devoted to the photoinduced birefringence have examined the phenomenon under a continuous wave irradiation obtained from gasor solid-state lasers $(488,514,532 \mathrm{~nm})$ [12]. Many of the 
Fig. 1 Experimental set-up for photoinduced birefringence measurements

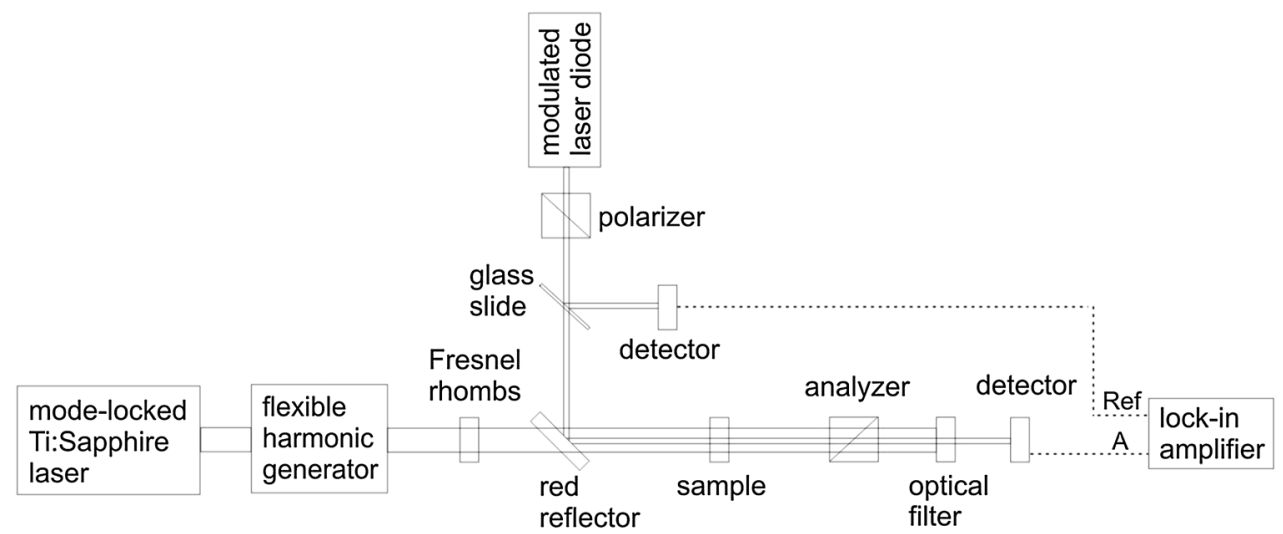

investigations have aimed at evaluating the structure-property relationships of different polymer materials at a fixed wavelength, which most frequently was located on the red side of the absorption band of trans azobenzene chromophores. On the contrary, the measurements performed as a function of wavelength have not been common, despite the fact that they may provide a very useful knowledge on the dominant photoisomerization pathway or the excitation conditions that ensure the fastest rate of the birefringence growth [13].

In this work, we examine the growth of the photoinduced birefringence in amorphous azobenzene-functionalized polymer at three excitation wavelengths. The material under study belongs to the polyimide family, which appears a very important for applications in photonics, due to a high glass transition temperature and an enhanced stability of the generated realignment [14]. Two of the applied excitation wavelengths have been located at exactly opposite sides of the trans azobenzene absorption band (i.e., 388 and $420 \mathrm{~nm}$ ). Third wavelength has been shifted to $438 \mathrm{~nm}$. To the best of our knowledge, the experimental studies of the photoinduced birefringence growths in azobenzene-derivative polymers under the irradiation of the wavelengths located symmetrically on the azopolymer film absorption band have not been reported so far.

\section{Experimental}

The synthesis and characterization of the investigated poly(esterimide) (PEI) was described in [15]. The polymer film was obtained from the cyclohexanone solution $(0.1 \mathrm{~g} / 1 \mathrm{~mL})$ spin coated onto glass substrate at $350 \mathrm{rpm}$. Drying was performed at $155{ }^{\circ} \mathrm{C}$ for $4 \mathrm{~h}$. The film thickness, measured by a Dektak XT stylus profiler, was $0.98 \mu \mathrm{m}$. The UV-Vis spectrum was recorded using a Perkin Elmer Lambda 40 spectrophotometer.

The sample birefringence was induced by the ultrashort-pulsed irradiation at the wavelengths of 388, 420 and $438 \mathrm{~nm}$. The beams were produced by a Flexible Harmonic Generator (GWU-FHG Spectra-Physics) as the second harmonics of a mode-locked Ti:Sapphire femtosecond laser (Tsunami, Spectra-Physics; time duration: $100 \mathrm{fs,}$ repetition rate: $80 \mathrm{MHz}$ ). The intensity of all the excitation beams was the same and equal to $70 \mathrm{~mW} / \mathrm{cm}^{2}$.

A weak, smaller in diameter, 690-nm beam from a laser diode was used for probing the induced birefringence changes in the material. The beam was modulated at $2580 \mathrm{~Hz}$. The excitation and probing beams were propagated co-linearly as shown in Fig. 1, being centered relatively to each other. The polymer film was placed perpendicularly into the optical pathway behind a red reflector used for combining both of the beams. The transmission of the probe beam through the crossed-polarizer set-up was detected by a silicon photodetector and measured using a lock-in amplifier at the probing beam modulation frequency. In order to obtain a maximum change in the transmission signal Fresnel rhoms were used to rotate the polarization of the writing beam so that it made an angle of $45^{\circ}$ with the horizontal polarization of the probing beam.

\section{Results and discussion}

The chemical structure of the investigated PEI together with the UV-Vis spectrum of the polymer film deposited on the glass slide is presented in Fig. 2.

The polymer possesses the covalently bonded chromophores with the fluorine atom substituent, attached to the main chain through a nitrogen atom. The absorption curve of the polymer film shows one band at $408 \mathrm{~nm}$ resulting from the overlap of a high intensity $\pi-\pi^{*}$ - and a lowintensity $n-\pi^{*}$ transitions of trans azochromophores [16]. On the basis of the absorption spectrum, the chromophores of the investigated polymer can be classified as push-pull azobenzenes.

The utilized harmonic generator combined with the mode-locked Ti:Sapphire 100-fs laser offered a unique 


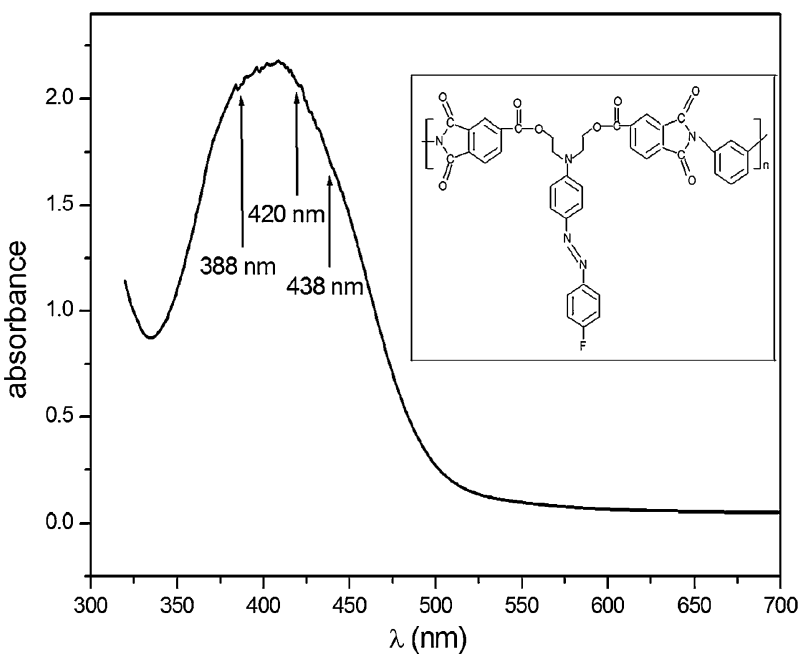

Fig. 2 UV-Vis spectrum of poly(esterimide) film with its chemical structure in the inset. The arrows indicate sample absorbance at the applied excitation wavelengths

possibility of a continuous change of the output wavelength by several tens of nanometers in the blue-violet spectral range. Therefore, for the investigated polymer, it was possible to tune two excitation wavelengths to the positions corresponding to the equal film absorbances (Fig. 2). The choice of the most blue-shifted wavelength was limited by the optics utilized in the experimental set-up. The film absorbance at a third excitation wavelength (i.e., $438 \mathrm{~nm}$ ) was $18 \%$ lower than that at 388 and $420 \mathrm{~nm}$.

The photoinduced birefringence was obtained from the measured transmittance $T$ through the crossed-polarizer set-up by the formula: $\Delta n=\lambda /(\pi d) \arcsin T^{1 / 2}$, where $\lambda$ is the probing beam wavelength and $d$ is the film thickness.

Figure $3 \mathrm{a}$ presents the birefringence curves recorded for the polymer film during ca. 10-min irradiation-the time period needed for achieving the saturation level in the case of 388-nm excitation.

The normalized birefringence relaxation curves recorded after turning off the excitation beams are shown in Fig. 3b. The most striking result is a large final birefringence generated upon illumination on the blue side of the chromophore absorption band, which almost doubles the final birefringence induced under irradiations at 420 and $438 \mathrm{~nm}$. Moreover, the birefringence relaxation observed after 388-nm illumination is slightly but distinguishably smaller than that observed after 420- or 438-nm irradiation.

In the functionalized polymer systems containing the azobenzene derivatives the birefringence growth with time is often described by the biexponential equation:

$\Delta n=A\left[1-\exp \left(-t / \tau_{A}\right)\right]+B\left[1-\exp \left(-t / \tau_{B}\right)\right]$,

where $\tau_{A}, \tau_{B}$ are the time constants and $A, B$ are the amplitudes associated with different physical processes
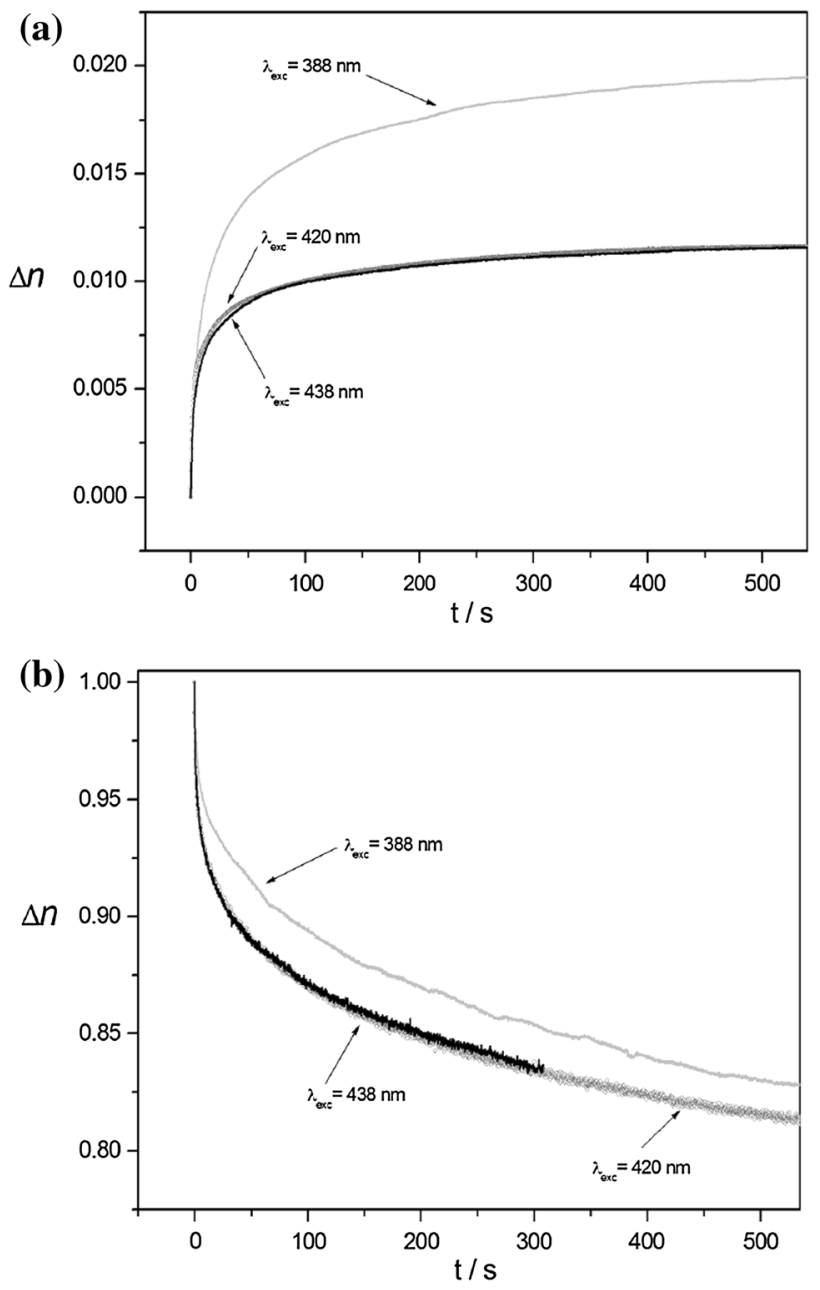

Fig. 3 Photoinduced birefringence of poly(esterimide) film after a turning on the 388-, 420- and 438-nm beams; $\mathbf{b}$ turning off the excitation beams

Table 1 Fitted parameters for Eq. (1)

\begin{tabular}{lllll}
\hline Excitation wavelength $(\mathrm{nm})$ & $A$ & $B$ & $\tau_{A}(\mathrm{~s})$ & $\tau_{B}(\mathrm{~s})$ \\
\hline 388 & 0.006 & 0.0094 & 3.0 & 26 \\
420 & 0.0054 & 0.0043 & 1.6 & 24 \\
438 & 0.0044 & 0.0051 & 1.5 & 21 \\
\hline
\end{tabular}

appearing upon illumination. According to Ho et al. [17] the fast $(A)$ and slow $(B)$ process could be associated with the reorientation of the azobenzene groups and the aligning motion of the polymeric segments, respectively. However, according to other authors, a different attribution of the processes may also be given [18, 19]. Although the attribution of the physical process appearing in the investigated PEI requires further studies, performing the curve fitting allowed us to quantitatively compare the obtained birefringence signals. The experimental data were analyzed within 
the initial $60 \mathrm{~s}$ of birefringence recording. After this time period, the birefringence reached the value of $81 \%$ of its maximum in the case of $420-\mathrm{nm}-$ and $74 \%$ in the case of $380-\mathrm{nm}$ excitation. The fitted parameters are collected in Table 1.

Comparison of the results obtained leads to the general features as follows:

1. Excitation on the blue and the red side of the absorption band results in two different time constants of the fastest process. The values of all the $\tau_{B}$ parameters are similar.

2. The highest $A$ and $B$ amplitudes are obtained for 388$\mathrm{nm}$ excitation. At this wavelength, the contribution $B /$ $(A+B)$ of a slower process to total birefringence is the largest.

The photoinduced birefringence in the polymer film is a consequence of the azobenzene chromophore realignment due to the trans-cis-trans isomerization cycles. The photoisomerization cycles involve two isomers, for which both the $\pi-\pi^{*}$ and $n-\pi^{*}$ transitions are possible. In the investigated material due to the overlap of $\pi-\pi^{*}$ and $n-\pi^{*}$ transitions of trans molecules, both of the transitions are excited during the laser irradiation with each wavelength. However, the $\pi-\pi *$ transitions are predominant ones [8].

Kim et al. [13] investigated a rate of the birefringence growth in azobenzene-derivative polymers while increasing the excitation wavelength along the red side of the trans isomer absorption band. The observed local maximum in the rate constant was correlated with cis-to-trans photoisomerization caused by absorption of the cis isomer in the $n-\pi^{*}$ band. In our study, by utilizing the irradiation wavelengths located on the opposite sides of the trans isomer absorption band and by exciting the same type of predominant transition for trans azochromophores, the effect of the cis absorption on the generated birefringence is probed.

For the 420- and 438-nm excitation wavelengths, the birefringence growth proceeds via the same photoisomerization pathway (i.e., the same types of transitions for trans and cis isomers) since both a very similar extent and dynamics of birefringence generation is observed. On the other hand, a different extent and dynamics of the birefringence growth upon the 388-nm irradiation in comparison with 420-nm one present an evidence of distinct isomerization pathways for the cis isomers (in either case, the pathways for trans-cis photoisomerization mainly involve the $\pi-\pi *$ transition). According to the earlier study [15], the absorption spectra of the investigated PEI in solution upon illumination by UV unpolarized light showed a new band at $368 \mathrm{~nm}$ assigned to the cis $-\pi-\pi^{*}$ transition. A cis $-n-\pi^{*}$ transition was not clearly revealed in that study. Taking the above into account, it can be assumed that the 388-nm irradiation stimulates the cis-to-trans transitions in the $\pi-\pi^{*}$ band. Since both the 420- and 438-nm wavelengths are located far from the maximum of cis- $\pi-\pi^{*}$ band it is reasonable to suspect that under these irradiation conditions the cis-to-trans photoisomerization occurs predominantly by the $n-\pi *$ transition of the cis isomer.

According to the quantum calculations performed by Pedersen et al., the optical storage performance of azocompounds with separated bands for cis isomers is improved when a more polarized $\pi-\pi^{*}$ band for cis isomers is used [20] i.e., at the excitation conditions for which a high degree of anisotropy of the cis isomers was revealed. This could be one of the factors responsible for the differences in the birefringence curves obtained at $388 \mathrm{~nm}$ than at $420 \mathrm{~nm}$ in our study. Besides, we believe that a stronger absorption of cis molecules in the $\pi-\pi^{*}$ band in comparison with the absorption in the $n-\pi^{*}$ band should account for a larger final birefringence generated at $388 \mathrm{~nm}$ than at $420 \mathrm{~nm}$. It is reasonable to suspect that a larger number of the cis-trans photoisomerization cycles induce larger segmental motions of the polymer chains, what could turn into a higher amplitude of the slow process observed in the sample.

The decay of birefringence after turning off the excitation light is caused by the thermal cis-trans isomerization of azochromophores and a thermal randomization of the molecular orientation [17]. A slightly larger birefringence relaxation after the irradiation on the red side of the absorption band than on the blue side might indicate a slightly higher number of cis isomers generated in the former case.

\section{Conclusions}

The photoinduced birefringence signals of azobenzene PEI film have been measured for the laser beams of the same intensities and the wavelengths located on the opposite sides of the trans azochromophore absorption band. The excitation on the blue side of the band induced twice larger final birefringence than the excitation on the red side however, with a slower dynamics. The absorption of cis isomer should be responsible for the differences of the generated birefringence signals, as one of the reasons.

Acknowledgments This study was supported by the Foundation for Polish Science under Grant No. POMOST/2013-7/6 co-financed from the European Union under the European Regional Development Fund.

Open Access This article is distributed under the terms of the Creative Commons Attribution License which permits any use, distribution, and reproduction in any medium, provided the original author(s) and the source are credited. 


\section{References}

1. A. Natansohn, P. Rochon, Chem. Rev. 102, 4139 (2002)

2. T. Todorov, L. Nikolova, N. Tomova, Appl. Opt. 23, 4309 (1984)

3. S. Wu, S. Duan, Z. Lei, W. Su, Z. Zhang, K. Wang, Q. Zhang, J. Mater. Chem. 20, 5202 (2010)

4. S. Hvilsted, C. Sanchez, R. Alcala, J. Mater. Chem. 19, 6641 (2009)

5. K.G. Yager, C.J. Barrett, J. Photochem. Photobiol. A 182, 250 (2006)

6. T. Alasaarela, D. Zheng, L. Huang, A. Priimagi, B. Bai, A. Tervonen, S. Honkanen, M. Kuittinen, J. Turunen, Opt. Lett. 36, 2411 (2011)

7. A. Emoto, E. Uchida, T. Fukuda, Polymers 4, 150 (2012)

8. H.M.D. Bandara, S.C. Burdette, Chem. Soc. Rev. 41, 1809 (2012)

9. M. Dumont, Z. Sekkat, Proc. SPIE 1774, 188 (1992)

10. O.V. Yaroshchuk, A.D. Kiselev, Y. Zakrevskyy, T. Bidna, J. Kelly, L.-C. Chien, J. Lindau, Phys. Rev. E 68, 011803 (2003)

11. Y. Atassi, J. Chauvin, J.A. Delaire, J-F. Delouis, I. Fanton-Maltey, K. Nakatani, Pure Appl. Chem. 70, 2157 (1998)
12. R.H.E Halabieh, O. Mermut, C.J. Barrett, Pure Appl. Chem. 76, 1445 (2004)

13. M.J. Kim, J. Lee, C. Chun, D.Y. Kim, S. Higuchi, T. Nakayama, Macromol. Chem. Phys. 208, 1753 (2007)

14. E. Schab-Balcerzak, M. Grucela, A. Kozanecka-Szmigiel, K. Switkowski, Opt. Mater. 34, 733 (2012)

15. E. Schab-Balcerzak, B. Sapich, R. Hass, J. Stumpe, Proc. SPIE 5724, 301 (2005)

16. H. Rau, Photochromism: Molecules and Systems (Elsevier, Amsterdam, 2003). (Revised edn.)

17. M.S. Ho, A. Natansohn, P. Rochon, Macromolecules 28, 6124 (1995)

18. O.K. Song, C.H. Wang, M.A. Pauley, Macromolecules 30, 6913 (1997)

19. Z. Sekkat, M. Dumont, Synth. Met. 54, 373 (1993)

20. T.G. Pedersen, P.M. Johansen, H.C. Pedersen, J. Opt. A Pure Appl. Opt. 2, 272 (2000) 